\title{
Psychiatrisierung, Personifizierung und Personalisierung Zur personenzentrierten Beratung in Organisationen ${ }^{1}$
}

\author{
Stefan Kühl, Hamburg
}

Bei Supervision und Coaching handelt es sich um die Beratung von Personen im Rahmen ihrer Tätigkeit in Organisationen. Die personenzentrierte Beratung von Organisationsmitgliedern hat schon den Entstehungsprozess moderner Organisationen begleitet. Das Gespräch mit Partnern und Freunden, mit denen man die kleinen Frustrationserlebnisse des Berufslebens aufzuarbeiten versucht, oder das Gespräch mit Kollegen bei der Rückfahrt von einer Dienstreise oder in einer Frühstückspause konnte den Charakter einer personenzentrierten Beratung annehmen. Auch das Vier-Augen-Gespräch mit dem Vorgesetzten konnte (oder kann) schon alleine durch sein Interaktionssetting häufig die Form einer Beratungssituation bekommen.

Die Besonderheit von personenzentrierter Beratung in der Form von Coaching und Supervision ist, dass sich für die Beratungssituation eine eigene Leistungsrolle ausdifferenziert. Die personenzentrierte Beratung wird nicht nebenbei von einem Lebenspartner, Freund, Kollegen oder Vorgesetzten durchgeführt, sondern durch einen speziell ausgebildeten Supervisor oder Coach. Dies führt dazu, dass sich die Beratungsinteraktionen nicht zufällig am Abendbrotstisch, in einer Kaffeepause oder in einem Mitarbeitergespräch ergeben, sondern im Rahmen einer genau benennbaren (weil notwendigerweise abrechenbaren) Zeitperiode stattfinden.

Bei der personenzentrierten Beratung geht es, so die Selbstbeschreibungen, primär um die „Sicherstellung“, „Ausweitung“ und „Optimierung" der beruflichen Arbeit der Klienten (vgl. Buchinger, Götz 2000, 34). Die personenzentrierte Beratung richtet sich an eine spezifische - in der Regel in einer Organisation tätige - Person. Häufig handelt es sich um Personen, die - wie im Fall der Supervision für Sozialarbeiter, Krankenschwestern oder Seelsorger - anspruchsvolle Leistungen am Klienten der Organisation erbringen oder die mit komplizierten hierarchischen oder lateralen Führungsaufgaben in

1 Eine ausführliche Fassung dieses Textes kann von der Website www.hsu-hh.de/kuehl heruntergeladen werden. Die dort verfügbare Fassung enthält eine stärkere wissenschaftliche Kontextualisierung des Arguments und eine in Fußnoten vorgenommene Erläuterung einzelner Argumentationsschritte. Auf der Website befinden sich auch weitere Texte zum Thema Coaching und Supervision. 
der Organisation betraut sind. Der Beratung wird in der Regel in einem zeitlich begrenzten, aus mehreren ein- bis dreistündigen Sitzungen bestehenden Prozess durch spezial ausgebildete Supervisoren oder Coaches erbracht. Die personenzentrierten Berater können in der Organisation fest angestellt sein. Häufiger handelt es sich jedoch um externe Berater, die über ein mehr oder minder formalisiertes Verfahren in einen Beraterpool der Organisation aufgenommen wurden (vgl. Rauen 2000, 303ff.; Fellermann 2003).

Supervision und Coaching unterscheidet sich in verschiedenen Aspekten von anderen, in der Literatur wesentlich prominenter behandelten Beratungsformen. Von der Organisationsberatung grenzen sich diese personenzentrierten Beratungsansätze insofern ab, als dass explizit auf die Rolle des einzelnen Mitarbeiters in der Organisation fokussiert wird (vgl. Buchinger, Götz 2000, 33ff.). Von der Psychoanalyse und der Psychotherapie lässt sich dieser Ansatz dadurch differenzieren, dass es nicht um die Person als Ganzes geht, sondern vorrangig um eine Rolle, um die Tätigkeit innerhalb von Organisationen (vgl. Rauen 2003; Schmidt-Lellek 2003). Vom Training, dem lange Zeit bevorzugten Instrument der Personalentwicklung in Organisationen, unterscheiden sich Supervision und Coaching - jedenfalls in ihren Ursprungsformen - vorrangig dadurch, dass die Beratung „unter vier Augen“" stattfindet. Zwar gibt es verschiedene Überlegungen zur Expansion der personenzentrierten Beratung in Richtung auf GruppenSupervision, Team-Coaching, Abteilungs-Coaching, Organisations-Supervision oder System-Coaching. Aber letztlich findet dann, so Wolfgang Looss $(1997,155)$, entweder eine „Einzelberatung unter Zeugen“ statt, oder die Beratungssituation „degeneriert“ zu einer Trainings- oder Seminarmaßnahme.

Wie in anderen Instrumenten der Personalentwicklung auch wird im Coaching und in der Supervision davon ausgegangen, dass die mit der ,individuellen Beratung" verbundenen personenzentrierten Ziele und die personalentwicklerischen organisationsbezogenen Ziele der "Steigerung der Effizienz" ineinander greifen. Bei personenzentrierter Beratung in Organisationen, so Astrid Schreyögg (2005, 160), könnten berufliche Selbstgestaltungspotenziale der einzelnen Person und die Optimierung der einzelnen Personen kombiniert werden. Oder anders ausgedrückt: Personenzentrierte Beratung stehe, so Kurt Buchinger und Klaus Götz $(2000,33)$, vor einer zweifachen Herausforderung: Es müssen ,wertschöpfende Beiträge zur Erreichung persönlicher Ziele der in der Organisation tätigenden Personen“ und gleichzeitig zur „Erreichung strategischer Ziele der Organisation“ geschaffen werden. Die „Personenentwicklung“, also die Entfaltung individueller Potenziale, korrelierte unmittelbar mit der „Personalentwicklung“, folglich der auf Ziele der Organisation ausgerichteten Optimierung des „Produktionsmittels Personal" (vgl. zur Begrifflichkeit Neuberger 1991, 14ff.; s. auch Schreyögg 2003, 220).

Die Quellen der Supervision als Reflexionsdisziplin der Sozialarbeit gehen bis ans Ende des 19. Jahrhunderts zurück (vgl. Belardi 2002, 133f.). Die Supervision hat aber in den meisten Ländern mit der Ausbildung einer ausdifferenzierten Berufsrolle des Supervisors erst in den letzten 30 Jahren eine breite Popularisierung erlebt. Coaching ist im Gegensatz zur Supervision ein neues Phänomen. Es ist als Beratungsprozess besonders für Führungskräfte in den 80er Jahren entstanden und erlebt eine große Popularität erst seit Ende der 90er Jahre (vgl. Böning 2002, 21).Während die personenzentrierte Beratung sich in Organisationen der sozialen Hilfe sehr früh unter dem Begriff der Supervision mit einer positiven Konnotation etablieren konnte, war die Inanspruchnahme in Unternehmen und Verwaltungen eher problematisch. Coaching wurde als Angebot für die „Schwächlinge“ unter den Führungskräften angesehen. Die Inanspruchnahme 
von personenbezogener Beratung fand unter größter Verschwiegenheit statt. Um die Assoziationen mit der Therapie zu vermeiden, wurde vielerorts Coaching unter Decknamen wie „Management-Sparring“ eingeführt. Inzwischen hat sich die Reputation von personenzentrierter Beratung auch in Unternehmen und Verwaltungen grundlegend verändert. Auch wenn Organisationen, in denen es ,schick“ ist, Coaching in Anspruch zu nehmen, eher die Ausnahme darstellen, so hat sich personenzentrierte Beratung doch als Personalentwicklungsmaßnahme in größeren Unternehmen, Verwaltungen, Krankenhäusern und sozialen Einrichtungen etabliert (vgl. Taffertshofer 2006).

Für die systemtheoretisch orientierte Organisationssoziologie ist dieser sich etablierende Beratungsansatz interessant, weil darüber das Verhältnis von Organisation und Person in den Fokus genommen werden kann. Es muss dabei genauer spezifiziert werden, unter welchen Gesichtspunkten Personen durch die Organisation, in der sie Mitglied sind, aber auch durch Beratungsfirmen, Berufsverbände oder andere Organisationen beobachtet werden. Es interessiert dabei also nicht das sich dem Instrumentarium der Soziologie entziehende psychische System und erst recht nicht der Mensch, sondern Personen, also die psychischen Systeme, die von sozialen Systemen (aber auch von anderen psychischen Systemen) beobachtet werden (Luhmann 1984, 155) und als „Adress- und Identifikationsstellen“ (Hohm 2006, 100) für die Organisationen dienen.

Ziel des Artikels ist es, am Beispiel der personenzentrierten Beratung die unterschiedlichen Formen von Personen als Adress- und Identifikationsstelle zu spezifizieren. Dabei werden drei verschiedene „Denkkonzepte“ durchgespielt und auf ihre Anwendbarkeit für das Phänomen des Coachings und der Supervision geprüft. Unter „Psychiatrisierung“ wird die teilweise Auflösung der für Organisationen charakteristischen Rollendifferenzierung verstanden. Es wird geprüft, ob durch personenzentrierte Beratung eine Integration weiterer, außerhalb der Organisation liegender Rollen stattfindet und es dadurch zur einer Kollabierung der Grenzen zwischen unterschiedlichen Rollen kommt (Abschnitt 1). Unter ,Personifizierung“" wird verstanden, dass in Organisationen viele Entscheidungen auf Personen zugerechnet werden. Organisationen können sich diesen Prozess insofern zunutze machen, indem sie über Einstellungen, Versetzungen, Entlassungen und Personalentwicklung Strukturen der Organisation prägen (Abschnitt 2). Unter ,Personalisierung“" wird der Prozess verstanden, auf die Kommunikations- und Entscheidungswege oder Konditional- und Zweckprogramm zurückgehende Konflikte als auf Personen zuzurechnende Konflikte zu behandeln und damit Organisationen von diesen Konflikten zu entlasten (Abschnitt 3). Das Verhältnis zwischen „Psychiatrisierung“, „Personifizierung" und „Personalisierung“ wird in jedem Beratungsprogramm einer Organisation, ja in jeder einzelnen Beratungssituation neu ausgehandelt. Soziologisch gibt es wenige Gründe dafür, die Funktion von personenzentrierter Beratung in der „Veränderung von Personen“ zu sehen. Vielmehr scheinen die Funktionen vorrangig in der für die Organisation vorteilhaften Isolierung der auf Personen zugerechneten Probleme in spezialisierten Interaktions-Settings zu liegen (Abschnitt 4).

\section{Psychiatrisierung}

In der Organisationssoziologie wurde die Organisation lange Zeit als weitgehend entpersonalisiertes System wahrgenommen. Diese in verschiedenen Strängen der Organisationssoziologie vertretene Auffassung geht auf Max Weber zurück, der in „Wirt- 
schaft und Gesellschaft“ behauptet, dass „,sachliche“ Unpersönlichkeit“ das Gebaren von Organisationen kennzeichne (Weber 1972, 578). Diese Ausrichtung auf Unpersönlichkeit mache es möglich, dass sich eine Organisation wie eine „Maschine“ verhält, die durch „Schnelligkeit, Eindeutigkeit, Aktenkundigkeit, Kontinuierlichkeit, Diskretion, Einheitlichkeit, straffe Unterordnung, Ersparnisse an Reibungen, sachlichen und persönlichen Kosten" gekennzeichnet seien (Weber 1972, 561f.).

Die im Gegensatz zu Max Weber kritisch gewendete Übernahme dieser Auffassung von Organisationen als entpersonalisierten Systemen ermöglichte dann, eine Konfliktsituation zwischen den mit Zwängen, Herrschaft und Entfremdung markierten Organisationen und den sich diesen Zwängen ausgesetzten Individuen aufzubauen. Man denke nur an die These Theodor W. Adornos (1954), dass die moderne Gesellschaft durch eine immer stärkere Durchdringung mit Organisationen gekennzeichnet sei, in denen sich das Menschliche kaum entwickeln könne. Oder man betrachte die Überlegungen von William H. Whyte (1956) über den „Organization Man“, der sich den strukturellen Mechanismen von Schulen, Universitäten, Unternehmen oder Verwaltungen anpasse und eine der Organisation angepasste Persönlichkeitsstruktur entwickle, die auch über die eigentliche Organisationsrolle hinaus prägend wirke.

Das Verständnis von Organisationen als entpersonalisierten Systemen diffundierte bis in Praktikerauffassungen in Organisationen. Man denke nur an die Diskussionsstränge in der Organisationsberatung, die Organisationen vorrangig von ihren Organisationsstrukturen aus betrachten. Diese Tendenz findet sich in der Expertenberatung à la McKinsey und Boston Consulting, in der es eine auffällige Spaltung in eine abstrakte Lobpreisung der Personen als „Humankapital“ und der vorrangig auf personale Passung zu den Organisationsstrukturen ausgerichteten Rationalisierungsmaßnahmen gibt.

Das Verständnis von Organisationen als entpersonalisierten Systemen gewinnt auf den ersten Blick ihre Berechtigung, wenn als ein zentrales Merkmal von Organisationen angesehen wird, dass diese nicht die komplette Person beinhalten, sondern lediglich ein Teil des Leistungsrepertoires abfragen. Damit unterscheiden sich Organisationen in der modernen Gesellschaft in einem zentralen Punkt von ansonsten auffällig strukturähnlichen Gebilden wie Gilden oder Klöster. In diesen Gebilden, die in der stratifizierten Gesellschaft dominierten, hatte und hat man es mit einer weitgehenden Totalinklusion der Leistungsträger zu tun. Gilden oder Klöster nehmen für sich in Anspruch, Lebensgemeinschaften zu sein und dementsprechend auch alle Rollen eines Mitglieds zu definieren (vgl. Kieser 1989).

Die Organisationen sind gegenüber ihren in der stratifizierten Gesellschaft dominierenden Vorläufern durch eine doppelte Ignoranz gekennzeichnet. Auf der einen Seite kann eine Organisation außerorganisatorische Anforderungen des Mitglieds an die Organisation zurückweisen. Die Frage um eine Gehaltserhöhung, weil man ein neues Haus gebaut hat, erscheint genauso illegitim wie die Bitte, doch nicht entlassen zu werden, weil man eine Großfamilie zu versorgen hat. Die Innehabung eines Amtes in einer Organisation kann nicht mehr wie im Mittelalter als „Besitz einer gegen Erfüllung bestimmter Leistungen ausbeutbaren Renten- oder Sportelquelle“ verstanden werden; vielmehr gelte bei Übernahme eines Amtes eine „spezifische Amtstreuepflicht gegen Gewährung einer gesicherten Existenz“ (Weber 1972, 553).

Auf der anderen Seite kann aber auch das Organisationsmitglied erwarten, dass seine anderen Rollen die Organisation nur insofern interessieren, als dass sie Rückwirkungen auf die Organisationsmitgliedschaft betreffen. Der Mitarbeiter eines Unternehmens oder eines Krankenhauses kann erwarten, dass die Mitgliedschaft in der CDU, die Her- 
kunft aus einem alten Adelsgeschlecht oder (bzw. und) die Leidenschaft für sadomasochistischen Sex von der Organisation ignoriert wird. Diese Entwicklung ist auch für die Organisation funktional, weil sie sich bei der Auswahl von Mitgliedern auf für sie „relevante Kriterien“ beschränken kann. Bei der Auswahl von Mitgliedern stehen anders als in stratifizierten Gesellschaften - universelle (und nicht partikulare), an Leistung orientierte (nicht askriptive) Kriterien im Mittelpunkt.

Die Konzentration auf die Organisationsrolle führt letztlich zu einer „Entpsychiatrisierung" der Organisation. Die Ehestreitigkeiten eines Mitarbeiters, die Schwierigkeiten eines türkischstämmigen Managers, die Erwartungen seiner Familie mit denen der Organisation vereinbar zu machen oder die privaten Vorlieben eines Auszubildenden für die Gothic-Szene müssen von der Organisation nicht als Problem begriffen werden. Es müssen für so geartete Probleme keine Stellen in der Organisation vorgehalten werden, die sich damit auseinandersetzen.

Ist der Boom von personenzentrierten Beratungen jetzt ein Indiz für eine Repsychiatrisierung der Organisation? Gibt es mit der Coaching-Welle ein neues Einfallstor für Therapeuten in die Organisationen? Wird der Mensch (mit all seinen Macken) wieder in den Mittelpunkt der Organisation gestellt? Auf den ersten Blick mag diese These eine gewisse Plausibilität haben. Die Bezeichnung von Coaching als „Couching“ gehört zu den bewährten Kalauern der Szene (vgl. Rauen 2000, 304). Der Widerstand gegen Coaching und Supervision, von dem gerade aus Unternehmen berichtet wird, erklärt sich auch aus den Erfahrungen, die mit gruppendynamischen Selbsterkenntnisprozessen in den 70er Jahren gemacht wurden (vgl. Schwertfeger 1998). Die Methoden, die in der Supervisions-Praxis angewendet werden, sind manchmal nicht grundlegend andere als die Methoden, die der Sozialarbeiter mit seiner Klientel einsetzt.

Auf den zweiten Blick scheint die These einer Repsychiatrisierung der Organisation jedoch nicht plausibel. Coaching und Supervision zeichnen sich im Gegensatz zu Psychotherapie und Psychoanalyse dadurch aus, dass sie alles andere als ein ganzheitlicher Ansatz sind. Es geht bei der Supervision und beim Coaching nicht um den Supervisand oder Coachee als ,ganzen Menschen“, sondern lediglich um seine Tätigkeiten in seiner Organisations- oder Berufsrolle. Bei der Betrachtung eines Menschen in seiner Berufsrolle mögen andere Rollen - als Familienmitglied, als Liebhaber oder als politisch Engagierter - mit einbezogen werden, sie interessieren aber nur als Hintergrund der Berufsrolle (vgl. Schreyögg 2002a, 40f.).

Deswegen wird bei Coaches und Supervisoren auch die Grenzziehung zwischen personenzentrierter Beratung in Organisationen und der personenzentrierten Therapie sehr genau thematisiert. Es herrscht - besonders angesichts der sonst fehlenden Standards in Coaching-Prozessen - eine überraschend große Klarheit darüber, welche Probleme im Rahmen eines Coaching-Prozesses oder einer Supervisionssitzung bearbeitet werden können und welche nicht. Die Frage der Überweisung von einem Coachee oder Supervisanden zu einem psychotherapeutischen Spezialisten wird als zentrale Fähigkeit eines personenzentrierten Beraters in der Organisation verstanden.

Wenn personenzentrierte Beratung also nicht als eine Repsychiatrisierung der Organisation verstanden werden kann, welche alternativen Erklärungen bieten sich dann an? 


\section{Personifizierung}

Es ist ein Verdienst Niklas Luhmanns, grundlegend mit dem Weberianischen Verständnis von Organisationen als entpersonalisierten Systemen gebrochen zu haben. Für Luhmann sind Personen nicht nur in Bezug auf die Passung zur Organisationsstruktur relevant, sondern sie sind selbst Element der Organisationsstruktur. Personen prägen nicht nur die Organisationsstrukturen und werden durch die Organisationsstrukturen geprägt, sondern sie werden in dem Luhmannschen Verständnis selbst als Organisationsstruktur verstanden. Luhmann ist mit diesem Verständnis in der Lage, eine symmetrisch aufgebaute Analyse von Organisationsstrukturen - von Entscheidungen, die als Prämissen für weitere Entscheidungen dienen - vorzunehmen. Er hält es für sinnvoll, in Organisationen drei verschiedene Formen von Entscheidungsprämissen zu unterscheiden (vgl. Luhmann 2000, 222ff.).

Der erste Typ von Entscheidungsprämissen sind Programme, die sich eine Organisation gibt. Diese können in Gesetzen, Policies, Verfahrensanweisungen oder Zielvereinbarungen schriftlich niedergelegt werden. Es gibt aber auch eine Vielzahl von Programmen, die nicht extra schriftlich fixiert sind, sondern lediglich verbal vermittelt werden. Luhmann unterscheidet zwei unterschiedliche Programme: In Zweckprogrammen werden Ziele vorgegeben, und das Organisationsmitglied ist frei in der Wahl der Mittel, um dieses Ziel zu erreichen. Die Zielvereinbarungssysteme in Organisationen sind Versuche, über die Vorgabe von Zwecken die Phantasie der Mitarbeiter in der Suche nach adäquaten Mitteln zu stimulieren. Bei Konditionalprogrammen handelt es sich um Wenn-dann-Regeln, die vorgeben, welchen Arbeitsschritt ein Mitarbeiter bei einem bestimmten Impuls zu erfüllen hat (a.a.O., 262ff.).

Der zweite Typ von Entscheidungsprämissen sind die Entscheidungs- und Kommunikationswege von Organisationen - und in der reflexiven Sprache Luhmanns die „Organisation der Organisation“ (a.a.O., 302ff.). Dabei wird festgelegt, wie Entscheidungen in Organisationen zu fällen sind und wie Kommunikationen zu verlaufen haben. Die bekannteste Form von Entscheidungs- und Kommunikationswegen sind die Hierarchien, die sich eine Organisation gibt. Diejenige, die durch ihre Position in der Hierarchie als zuständig angesehen wird, kann davon ausgehen, dass ihre Entscheidungskompetenzen nicht angezweifelt werden und ihre Entscheidungen auch dementsprechend umgesetzt werden. Neben der Hierarchie existieren aber auch noch andere Formen der Strukturierung von Entscheidungs- und Kommunikationswegen: die Mitzeichnungsrechte von Abteilungsleitern, die lateralen Entscheidungsstrukturen in Vorständen oder die basisdemokratisch organisierten Abstimmungen in Vereinen.

Als dritten Typ von Entscheidungsprämissen nennt Luhmann das Personal. Es mache, so Luhmann, einen Unterschied aus, ob Entscheidungen in einer Organisation durch einen Juristen, Betriebswirt oder Ingenieur getroffen werden. Die Ausrichtung von Entscheidungen unterscheidet sich, je nachdem ob jemand von außen in die Organisation kommt oder ob jemand bereits in der Organisation sozialisiert wurde (a.a.O., 279).

Stellen in der Organisation werden jetzt unterschiedlich stark durch diese drei Entscheidungsprämissen strukturiert. Eine Stelle am maschinellen oder administrativen Fließband einer Organisation basiert vorrangig auf einer Konditionalprogrammierung. Eine Stelle im mittleren Management ist sehr häufig ganz besonders durch ihre „Sandwich-Position" zwischen Vorgesetzten und Untergebenen, also durch die Einbindung in die Kommunikationswege geprägt. Stellen an der Spitze der Organisation fordern be- 
sonders die Entscheidungsprämisse Personal, weil die obersten Vorgesetztenpositionen nur sehr begrenzt durch die Programme und Kommunikationswege geprägt werden. Hier findet also - sichtbar an den äußerst aufwändigen Auswahlverfahren für Spitzenpositionen - eine vergleichsweise starke Form von Personifizierung statt.

Durch Luhmann ist das übergreifende Ordnungsschema für Organisationsstrukturen überzeugend herausgearbeitet worden. Es fällt jedoch auf, dass er eine weitere Auffächerung der Strukturtypen nur für Programme vorgenommen hat. Inspiriert durch die Vorlagen von Herbert Simon und James March wird eine sowohl theoretisch als auch empirisch fruchtbare Unterscheidung in Zweck- und Konditionalprogramme vorgenommen (vgl. früh schon Luhmann 1964b; 1966a). Für die Entscheidungsprämissen „Entscheidungs- und Kommunikationswege“ und für die Entscheidungsprämisse für „Personal" finden wir bisher jedoch nicht eine vergleichbare Aufschlüsselung.

Im Folgenden wird vorgeschlagen, die Einstellung, die Versetzung, die Entlassung und die Personalentwicklung als verschiedene Strategien zu betrachten, mit denen Organisationen an ihrem Strukturtypus Personal arbeiten können. In der Organisation gibt es, anders ausgedrückt, verschiedene Möglichkeiten, an der Strukturkomponente Personal zu arbeiten: durch die Frage, wer eingestellt wird, wer entlassen wird, wer wie versetzt wird und wer durch Personalentwicklungsmaßnahmen neu ausgerichtet wird.

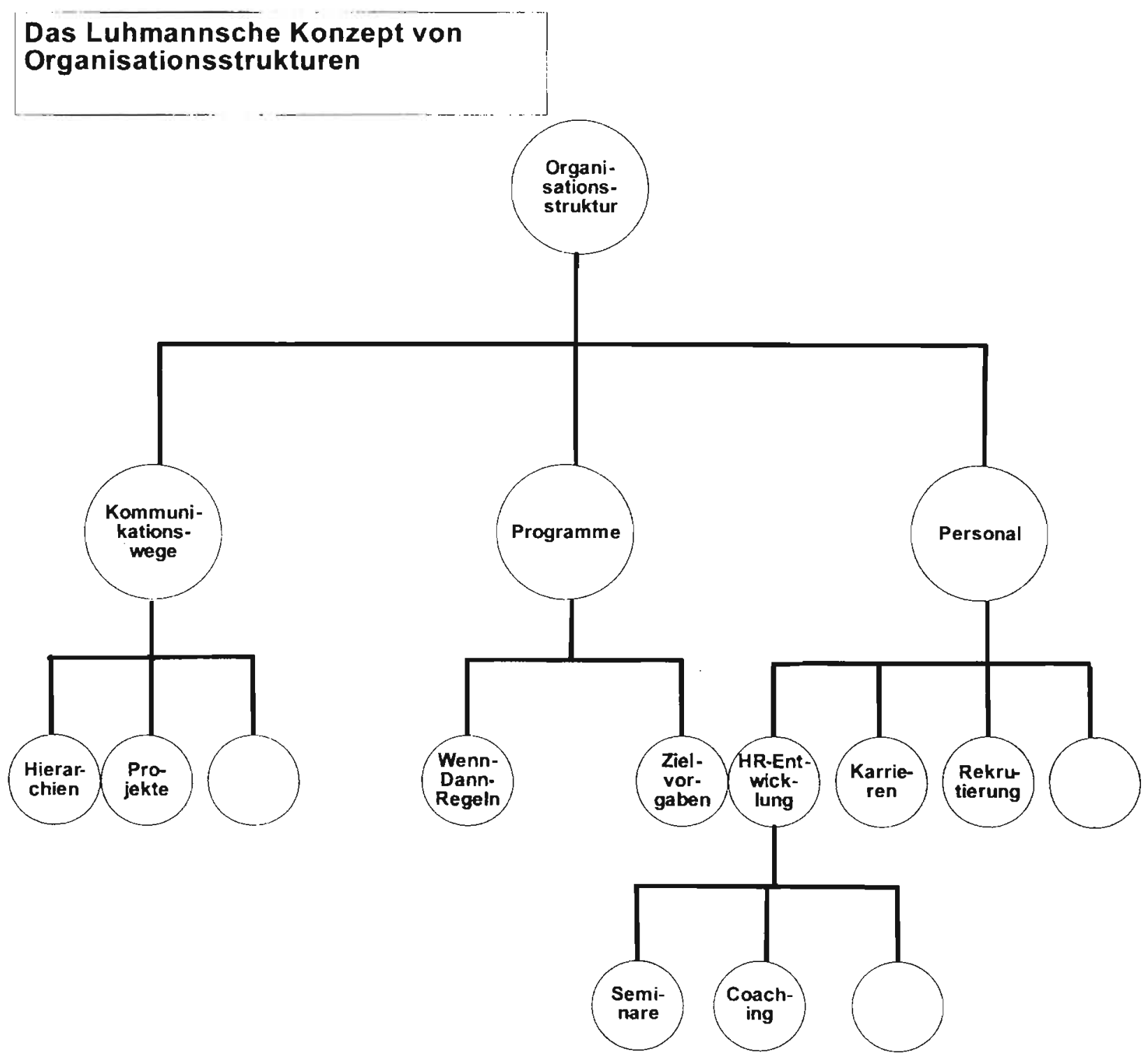


Die genannten vier Kategorien lassen sich zwar in allen Organisationen vorfinden, unterliegen aber jeweils spezifischen Beschränkungen: Bei der Einstellung kann eine Organisation der Einschränkung ausgesetzt sein, dass nur ein vorher fixierter Lohn bezahlt werden kann und so der vielleicht geeignet erscheinende Bewerber nicht gelockt werden kann. Die Versetzung von Mitarbeitern ist häufig Begrenzungen unterworfen, weil diese erst durch das mikropolitische Netz in Organisationen gepresst werden müssen. Entlassungen - und damit die Möglichkeit zur Neueinstellung - sind häufig alleine schon aufgrund arbeitsrechtlicher Beschränkungen kompliziert, wenn nicht sogar gänzlich unmöglich. Personalentwicklungsmaßnahmen müssen in der Regel durch den Betriebsrat genehmigt werden und scheitern nicht selten an dem Widerstand der Abteilungen, die für die Kosten aufzukommen haben.

Vor dem Hintergrund dieser Beschränkungen lassen sich die verschiedenen Kategorien der Entscheidungsprämisse Personal als funktionale Äquivalente behandeln. Wenn die Organisation aufgrund von Budgetbeschränkungen nicht zur Einstellung neuen Personals in der Lage ist, wird - wie das Beispiel von Ministerien nach Regierungswechseln zeigt - durch Versetzungen versucht, neue Entscheidungssituationen herzustellen. Wenn eine Organisation -wie zum Beispiel im Falle einiger öffentlicher Verwaltungen - durch die weitgehende Verhinderung von Entlassungen und Versetzungen hier keine Strukturflexibilität hat, kann versucht werden, durch Personalentwicklungsmaßnahmen eine andere Form der Entscheidungsfindung sicherzustellen.

Die vier Kategorien der Entscheidungsprämisse Personal lassen sich jeweils wiederum untergliedern. So lassen sich organisationsinterne gruppenbasierte Trainings, die Aufstellung von Lernprojekten, die Teilnahme an externen Fortbildungsmaßnahmen und eben personenzentrierte Beratung als verschiedene Ausprägungsformen von Personalentwicklung begreifen. Lässt sich aus dieser Betonung von Personal auch eine Betonung von Personalentwicklung ableiten? Ist Luhmann vielleicht ein bisher verkannter Promotor der Personalentwicklung?

Personalentwicklung ist eine Möglichkeit, den Strukturtypus Personal zu verändern, aber nach Auffassung von Luhmann im Vergleich zu den funktionalen Äquivalenten Einstellung, Versetzung oder Entlassung eine relativ schwache. Dies hängt mit der von Luhmann vertretenen, der Alltagsauffassung entgegengesetzte Betrachtung der Wandlungsfähigkeit von Personen und Organisationen zusammen. Im Vergleich zu Personen sind, so die überraschende These, Organisationen geradezu ein Wunder der Veränderungsfähigkeit. Kommunikations- und Entscheidungswege, Zweck- und Konditionalprogramme und die Zuordnung von Personen zu Stellen können häufig durch eine einfache Entscheidung geändert werden, während sich die Veränderbarkeit der Person sich dieser Entscheidbarkeit entzieht.

Dies hängt mit dem Charakter von Personen als ,individuell attribuierte Einschränkung von Verhaltensmöglichkeiten" zusammen. Personen sind - anders als Programme und Kommunikationswege - nicht weiter dekomponierbar. Ein Zweckprogramm zur Eroberung des Bohrerkassettenmarktes in Polen kann weiter in eine Vielzahl von Unterprogrammen zerlegt werden. Ein Kommunikationsweg kann allein durch die Zwischenschaltung einer weiteren Managementebene noch weiter aufgefächert werden. Dieser Mechanismus der Dekomposition funktioniert bei Personen nicht. Personen sind - Michel Houellebecq (2001) paraphrasierend - soziologisch gesprochen „Elementarteilchen", eine nicht weiter dekomponierbare soziale Einheit.

Personen können deswegen von Luhmann als Lösungen für das Problem der doppelten Kontingenz von sozialen Systemen verstanden werden. Nur bei Erwartungsdis- 
ziplin und bei Einschränkung könnten Personen helfen, die ,instabile, zirkuläre Notlage der doppelten Kontingenz" zu überwinden (Luhmann 2005, 142f.). Würde eine Person nicht versuchen, gegenüber anderen Personen oder sozialen Systemen die zu bleiben, die man vorgetäuscht hat zu sein, würde sie keinen Beitrag zur Kontrolle des Problems der doppelten Kontingenz liefern.

Das Besondere von Personen im Vergleich zu Organisationen ist, dass sie sich nicht durch eine Änderung der Selbsterwartungen allein verändern lassen. Selbst wenn die einzelne Person bereit wäre, sich zu ändern, ist sie durch die sozialen Erwartungen festgelegt, mit denen sie täglich durch andere Personen und soziale Systeme konfrontiert wird. Der eigentlich geläuterte aus der Haft entlassene Kriminelle, der wegen seiner Rückkehr in das alte Milieu wieder straffällig wird, und das alkoholkranke Familienmitglied, das durch die Familie ungewollt in ihrem Suchtverhalten bestärkt wird, sind nur die prominentesten Figuren für die Veränderungsschwierigkeiten bei Personen. Aber auch in der Organisationsforschung gibt es dafür vielfältige Beschreibungen. Man denke nur an den Manager, der am Montag voller Tatendrang von einem Wochenendseminar zurückkommt und von seinen Vorgesetzten, Kollegen und Untergebenen in einer eigenen Art von 360-Grad-Feedback (,Du bist plötzlich so anders“) innerhalb kürzester Zeit ausgekühlt wird.

Aber auch veränderte Fremderwartungen reichen nicht aus, Personen zu verändern. Zwar kann es sein, dass der Ehemann aufgrund des Drucks seiner Frau haushälterische Interessen heuchelt oder dass ein Mitarbeiter angesichts des demokratischen Führungsstils seiner Chefin die Partizipationsprosa bedient. Dies bleibt aber äußerst instabil, solange sich diese Veränderung auf die Fremderwartung ausrichtet und nicht durch Selbsterwartungen gestützt wird.

Die Person ist für Luhmann $(2000,280)$ ein ,Agglomerat von Selbsterwartungen und Fremderwartungen“, das sich einer Änderung nur der Selbst- oder nur der Fremderwartung entzieht. Diese Problematik wird unter dem Begriff auch in der Literatur über Personalentwicklung thematisiert. Gerade die Propagierung von personenzentrierter Beratung in Organisationen läuft über das Argument, dass die Veränderung von Selbst- und Fremderwartungen besser als zum Beispiel bei Trainings synchronisiert werden kann. Bei Supervisions- und Coachingmaßnahmen könnten beispielsweise Einzelberatungen systematisch an Beurteilungen durch Vorgesetzte, Kollegen und Untergebene rückgebunden werden. Auch sei es möglich, durch die Regelmäßigkeit der Sitzungen ein Abgleiten in alte Verhaltensmuster zu bearbeiten. Die Frage ist jedoch, ob sich eine solche kombinierte Veränderung von Selbst- und Fremderwartung nicht lediglich eine Planungsphantasie ist. Personales Gedächtnis (die Selbsterwartungen) und soziales Gedächtnis (die Fremderwartungen) seien, so Luhmann (ebd.), so miteinander „verfilzt“, dass eine planmäßige Änderung kaum möglich erscheine.

\section{Personalisierung}

Konflikte können in Organisationen auf zweifache Weise zugerechnet werden: als Konflikt zwischen Rollen oder als Konflikt zwischen Personen. Konflikte zwischen Rollen werden dabei organisationsintern als legitime Auseinandersetzungen gehandhabt. Hinter dem Konflikt zwischen Rollenträgern wird ein Organisationskonflikt gesehen. Es wird deswegen organisationsintern in der Regel akzeptiert, dass sich in der Universität die Fachgruppensprecherin Politologie mit dem Fachgruppensprecher Be- 
triebswirtschaftslehre über die Einbeziehung von wissenschaftlichen Mitarbeitern in die Lehre streitet. Konflikte zwischen Personen haben in Organisationen nicht die gleiche Form von Legitimität. Finden diese statt, werden sie als „Hahnenkämpfe“ zwischen Verwaltungsgockeln, als „Zickenkrieg“ zwischen mehreren Unteroffizierinnen oder als Problem in der "Chemie" zwischen zwei Abteilungsleitern markiert und tendenziell delegitimiert.

$\mathrm{Ob}$ eine Auseinandersetzung jetzt als Konflikt zwischen Rollen oder Konflikt zwischen Personen gehandhabt wird ist nicht durch die Organisation selbst objektiv bestimmbar, sondern wird sozial ausgehandelt. Es ist ein häufig zu beobachtendes Phänomen, dass in einer Interaktion eine Aushandlung darüber gesucht wird, ob ein Konflikt als „Chemie-Problem" bestimmt wird, das durch ein Konflikt-Coaching oder die Auswechslung eines der Beteiligten zu lösen wäre, oder ob es sich um einen Rollenkonflikt handelt, der auch bei der Auswechslung der Person bestehen bleiben würde.

In Organisationen gibt es, so jedenfalls die Beschreibung in der Soziologie, eine breite Tendenz, zu einer personalen Zurechnung zu neigen. Diese Problematik ist unter dem Stichwort der „Personalisierung" in der soziologischen Literatur aus verschiedenen Theorieperspektiven ausgearbeitet worden: Man neigt im Alltagserleben dazu, Schwierigkeiten, Spannungen und Enttäuschungen auf beteiligte Personen zurückzuführen (Luhmann 1962, 16). Irgendjemand habe Schuld, weil er „ehrgeizig, selbstsüchtig, faul oder eitel“ ist, oder er wird „als unfähig angesehen, weil er gewisse Erfahrungen nicht erfüllt".

Interessant ist, dass wir es in der Selbstbeschreibung des sozialen Systems mit einer Aneinanderreihung von kausalen Zurechnungen zu tun haben (,jemand ist Schuld"). Von einer Außenperspektive kann man dann aber eine konkurrierende Beschreibung anfertigen, in dem die „Personalisierung“ einer Schwierigkeit, Spannung oder Enttäuschung nicht mit dem konkreten Verhalten, sondern mit der sozialen Position des Beschuldigten zu erklären ist. Dieser Prozess der Zurechnung lässt sich, wie Réne Girad (1982) an der Funktion der Sündenböcke gezeigt hat, für die Gesamtgesellschaft beobachten. Sie findet aber in einer ähnlichen Form auch in Organisationen statt. Solche $\mathrm{Zu}$ rechnungen sind, so Luhmann $(1962,16)$, zumeist befriedigend - und man kann ergänzen, auch funktional. Und zwar, weil sie eine wichtige Entlastungsfunktion in Organisationen haben. Weil Personen in Organisationen so leicht greifbar sind, können diese für Fehler verantwortlich gemacht werden. Personen wird die Verantwortung für eine bestimmte Situation angelastet und durch die persönliche Zuordnung der Fehler weggedrückt. Die Person nimmt die Verantwortung auf ihre Kappe und entlastet damit die Organisation auf der häufig blockierenden Suche nach anderen Fehlerquellen (vgl. Brunsson 1989, 20f.).

Die organisationssoziologische Diskussion über Personalisierung bezog sich bisher fast ausschließlich auf weitgehend ungeplante Prozesse der personalen Zurechnung. Es ging um die mikropolitische Verschiebung von Problemen auf einzelne Personen (vgl. Jackall 1988), die ungewollt eingehandelten Lernschwächen (Weltz 1986) oder die latente Funktionalität dieser Personalisierung für die Organisation (Luhmann 1964). Das Phänomen Supervision und Coaching ist insofern interessant, weil sich hier eine Institutionalisierung dieser Personalisierungsfunktion in Organisationen andeutet. Über Supervision und Coaching werden strukturelle Konflikte personell zugerechnet und in gesonderte Besprechungssituationen abgelegt. Die Probleme der Mitarbeiter mit und in der Organisation werden durch die personenzentrierte Beratung in einen spezialisierten Kommunikationsbereich überwiesen. Die Supervisorin pflegt die „Psychohygiene“ in 
der Organisation, indem sie ein offenes Ohr für die Sorgen des Sozialarbeiters hat. Der Coach wird, salopp ausgedrückt, zu einem „Kummerkasten“, in dem das Organisationsmitglied seine Sorgen einwerfen kann.

Wie diese Prozesse der Personalisierung im Einzelnen ablaufen können, ist bisher lediglich für die Supervision im therapeutischen Bereich untersucht worden. Das Programm eines Krankenhauses sieht vor, so die Analyse von Ulrich Oevermann (1993; 2001), dass magersüchtige Patienten maximal fünfundzwanzig Sitzungen mit einem Therapeuten verbringen. Die Handhabung dieser Regelung führt zu Strukturproblemen, die sich besonders im Verhältnis des Therapeuten zu den Klienten äußern. Durch eine Fallsupervision wird dieses Strukturproblem jedoch personalisiert. Es wird in den Supervisionssitzungen als ein Problem von „Übertragung“ und „Gegenübertragung“ zwischen Patienten und Klienten thematisiert und die Organisation damit tendenziell von der Diskussion des strukturellen Konflikts entlastet.

Man darf diese Funktion personenzentrierter Beratung nicht unterschätzen. Sie kann gerade in einer Phase, in der die Organisation durch Veränderungsanforderungen überlastet ist, einen wichtigen Strukturschutz bilden. Die eigentlich durch die Organisation bedingten Probleme können als persönliche Probleme der Mitarbeiter in speziellen Räumen bearbeitet werden. Die Standardprozesse der Organisation werden dadurch von den aus der Organisation geborenen persönlichen Nöten und Leiden ihrer Mitarbeiter entlastet.

Aber gerade in dieser Funktion liegt auch die Gefahr. Die Isolierung der Konflikte in speziellen Interaktionssituationen kann, so die organisationstheoretisch nahe liegende Vermutung, dazu führen, dass die Reibungen, Konflikte und Auseinandersetzungen der Organisation als Material entzogen werden (vgl. Kieserling 1993, 26). Mit der Zurechnung auf eine Person wird die problematische Erfahrung nicht verarbeitet, sondern kaschiert. Negative Erfahrungen werden, so Friedrich Weltz, in Form von Kaltstellungen personenzentriert, nicht aber institutionell verarbeitet: Die Organisation wird, so die Zuspitzung von Weltz $(1986,533)$, aus dem „Schaden dumm“. Dieses Problem wird in der Supervision und im Coaching indirekt unter dem Label „Verhältnis von personenzentrierter Beratung und Organisationsentwicklung" angesprochen. Es setzen zunehmend Suchbewegungen ein, wie die in der personenzentrierten Beratung generierten Informationen an die Organisation angeschlossen werden können.

\section{Ausblick}

In der personenzentrierten Beratung in Organisationen können alle drei beschriebenen Phänomene - Psychiatrisierung, Personifizierung und Personalisierung - vorkommen. Das Verhältnis zwischen diesen Umgangsformen wird auf unterschiedlichen Ebenen thematisiert: bei der Formulierung eines für die Organisation verbindlichen personenzentrierten Beratungsprogramms, in dem die generalisierten Ziele, Auswahlregeln und Durchführungsbedingungen der personenzentrierten Beratung festgelegt werden; bei der Aushandlung eines konkreten Beratungs-Settings zwischen Personalentwickler, Berater und Klient und bei jeder einzelnen Sitzungen zwischen Berater und Klient.

Wie gezeigt, ist organisationssoziologisch eine Skepsis gegenüber der in Selbstbeschreibungen zu findenden Funktion von personenzentrierter Beratung angebracht. Die Versuche, über Supervision oder Coaching gezielt Personen und damit auch Strukturen der Organisationen zu verändern, mögen Erfolg versprechender sein als beim Training, 
aber auch bei einer sehr sorgfältigen Verknüpfung der Veränderung von Selbst- und Fremdwahrnehmung einer Person wird sich die Veränderungsresistenz von Personen aufgrund einer Verflechtung von personalem und sozialem Gedächtnis kaum überwinden lassen.

Diese systemtheoretisch begründete Skepsis gegenüber der Hebelwirkung von Supervision und Coaching stellt aber nicht die Funktionalität grundsätzlich in Frage, bezieht sie sich doch lediglich auf diese offensichtliche, manifeste Funktion. Die Funktion von personenzentrierter Beratung liegt jedoch, so der Vorschlag in diesem Artikel, weniger in der manifesten Funktion der Personalentwicklung denn in der latenten, versteckten Funktion der Isolierung von Konflikten in speziellen Interaktionszusammenhängen. Diese latente Funktion kann zunehmend wichtiger werden - und sich mittelfristig auch als manifeste, weil kommunikationsfähige Funktion ausbilden.

Personenzentrierte Beratung könnte sich dabei als ein funktionales Äquivalent zu den „Konfliktisolierungen“" etablieren, die sich in jeder Organisation im Wildwuchs, quasi „,von unten“ ausbilden. Das Ablästern bei Kollegen, die kleine Tuschelei am Rande eines Workshops oder die Sprüche an den Klos können die Funktion haben, Konflikte interaktionell zu isolieren. Der Reiz von personenzentrierter Beratung besteht darin, dass die Organisation mit der personenzentrierten Beratung diese Isolierung von Konflikten auf der Informalität wieder stärker in die Formalität zieht - vielleicht auch mit der Hoffnung, die Kontrolle über das Konfliktmaterial durch die Einbeziehung von Beratern nicht völlig zu verlieren.

Mit den Stichworten „Ambivalenz“ und „Anbau“ konstatiert Luhmann - sicherlich in Übereinstimmung mit einer Reihe anderer soziologischer Zeitdiagnosen - in Organisationen eine zunehmende Belastung der Strukturkategorie „Personal“. Ambivalenz bezeichnet dabei eine Unklarheit von Aufgabenzuweisung und Verantwortung. Durch eine „Entformalisierung“ und „Entbürokratisierung“ seien die Mitglieder der Organisation nicht mehr sicher, wie weit ihre Pflichten gehen. Genau dies mache sie, so Luhmann, aufnahmebereit für neuartiges Verhalten. Die „Grenzen der ablehnbaren Zumutungen“ würden verschwimmen. Damit verschwände auch die Sicherheit, die einmal darin gelegen hätte, dass man die Minimalbedingungen der Mitgliedschaft genau kannte. Diese ginge auf Kosten der Person: „Emotionale Anspannung“, „,kompensationsbedürftige Überbeanspruchung“ und „,angstbedingte Reaktionsbereitschaft“ könnten die Folge sein (Luhmann 1964, 151). Anbau bezeichnet demgegenüber eine andere Umgangsform mit neuartigen Beanspruchungen in Organisationen. Die Anpassung der Organisation fände, so Luhmann, durch den Anbau, durch eine „Erweiterung des Systems“ statt. Alles bliebe beim Alten, lediglich neue Stellen, neue Kontaktpersonen würden mit den Aufgaben betraut werden. Effekt sei dann jedoch das „Anschwellen interner Kommunikation und Konflikte“. Da die neuen Stellen nicht ohne Zusammenhang mit der bisherigen Organisation arbeiten könnten, müssten sie ihre Arbeitsweise mit den etablierten Stellen abstimmen. Die „Anpassung an Umweltveränderungen“, die durch die „Vergrößerung des Systems scheinbar gelöst werden würde", tauche als neuartiges Problem in der „Form von internen Konflikten wieder auf" (a.a.O., 149f.).

Für Luhmann (a.a.O., 152) sind die Ambivalenz- und die Anbaustrategie funktionale Äquivalente mit je eigenen Vor- und Nachteilen - für unseren Zweck ist jedoch nur wichtig, dass beide Strategien tendenziell auf „Kosten der Person“ gehen (um den Luhmannschen Ausdruck zu nehmen). Der Mitarbeiter, der sich in dem durch Korruption gekennzeichneten Land bewegt, entwickelt Gewissensbisse, wenn er den Verkaufsprozess durch kleine Geschenke beschleunigt. Die Automobilentwickler nehmen die 
Auseinandersetzung mit den Marketing-Experten persönlich und verbeißen sich in einen ,,an die Substanz gehenden“ Konflikt. Der Sozialarbeiter verzweifelt an seinen Jugendlichen, verbringt schlaflose Nächte und verliert deswegen an Lebensfreude.

Diese „Kosten der Person“ könnten der Organisation egal sein. Die Belastung der Person fällt in die „Indifferenzzone“ (Barnard 1938, 167ff.), die ein Organisationsmitglied akzeptieren muss. Sie werden jedoch für die Organisation dann relevant, wenn die Person die Belastung nicht bewältigen kann. Die Gewissensbisse lassen den Mitarbeiter in entscheidenden Verhandlungssituationen mit Kunden den Biss missen lassen. Die Entwickler übersehen bei der Personalisierung des Konflikts mit ihren MarketingKollegen zentrale Sachaspekte. Der Sozialarbeiter ist ausgebrannt und kann den Jugendlichen nicht mehr den Halt geben, die der Situation angemessen ist.

Organisationen müssen angesichts der wachsenden Belastung der Kategorie Person zusätzliche "Motivations- und Sicherheitsquellen" erschließen: z.B. innere Verpflichtung auf einen gemeinsamen Zweck oder den Aufbau von besonders intensiven Gruppensolidaritäten. Diese hier beispielhaft genannten Strategien brächten jedoch Kosten für die. Organisation mit sich, weil durch die Orientierung auf einen Zweck oder durch die Verpflichtung auf Gruppensolidarität die Organisation tendenziell ,entflexibilisiert". Supervision und Coaching können als zusätzliche Motivations- und Sicherheitsquellen verstanden werden, durch die Flexibilitätsnachteile durch Zweckidentifizierung oder Gruppensolidarisierung vermieden werden könnten.

\section{Zusammenfassung}

Unter den Begriffen der Supervision und des Coachings hat die personenzentrierte Beratung in Organisationen ein hohes $\mathrm{Ma}$ an Popularität erlangt. Das Ziel dieses Artikels ist es, unter Rückgriff auf die Systemtheorie die Funktion von personenzentrierten Beratungsansätzen wie Supervision und Coaching für die Organisation näher zu bestimmen. Es spricht einiges dafür, dass die Funktion von personenzentrierter Beratung weniger in der offensichtlichen, manifesten Funktion der Personalentwicklung liegt als in der versteckten, latenten Funktion der Isolierung von Konflikten in speziellen Interaktionszusammenhängen. Die Organisation erreicht durch Supervision und Coaching einen Schutz ihrer Strukturen, weil Konflikte interaktionell isoliert werden können und so für die Organisation weitgehend folgenlos bleiben.

Schlüsselbegriffe: Supervision, Coaching, Organisation, Systemtheorie.

Abstract: Psychiatrization, personification and personalization. Individual-centred consulting in organizations

The individual-centred consulting already achieved high popularity in supervision and coaching. Based on system theory this article aims to define the functions of individual-centred consulting approaches - such as supervision and consulting - for organizations. The function of person-centred consulting is less an obvious manifest function of human resources development and more an invisible one for isolating conflicts in special interactional contexts. The organization achieves protection of its structure through coaching and supervision, because it is able to isolate its conflicts interactionally and therefore these conflicts remain to a large extent without any consequences for the organization.

Key words: Supervision, coaching, organization, system theory.

\section{Literatur}

Adorno, T.W. (1954): Individuum und Organisation. In: Neumark, F. (Hg.): Individuum und Organisation. Darmstädter Gespräche. Dortmund: Neue Darmstädter Verlagsanstalt, S. 21-35. 
Barnard, C.I. (1938): The Functions of the Executive. Cambridge, MA: Harvard University Press. Belardi, N. (1992): Supervision. Von der Praxisberatung zur Organisationsentwicklung. Paderborn: Junfermann.

Beumer, U. (2003): Coaching und Supervision - Modelle zur professionellen Kompetenzentwicklung. Pädagogischer Blick 11, 144-154.

Böning, U. (2002): Der Siegeszug eines Personalentwicklungs-Instruments. Eine 10-Jahres- Bilanz. In. Rauen, C. (Hg.): Handbuch Coaching (2. Aufl.). Göttingen et al: Verlag für Angewandte Psychologie, S. 21-45.

Brunsson, N. (1989): The Organization of Hypocrisy: Talk, Decisions and Actions in Organization. Chichester: John Wiley and Sons.

Buchinger, K., Götz, K. (2000): Coaching. Ausgangspunkte, Gegenstände und Methoden. In: Götz, K. (Hg.): Human Resource Development. Band 2. München, Mering: Hampp, S. 3352.

Fellermann, J. (2003): Supervision. In: Handbuch Personalentwicklung. Köln: Luchterhand., 87. Erg. Lfg. 2.A1.

Girard, R. (1982): Le bouc émissaire. Paris: Grasset.

Goffman, E. (1961): Asylums. New York: Double Day.

Hohm, H.-J. (2006): Soziale Systeme, Kommunikation, Mensch (2. Aufl.). Weinheim: Juventa.

Houellebecq, M. (2001): Elementarteilchen. München: Econ.

JackalI, R. (1988): Moral Mazes. The World of Corporate Managers. New York; Oxford: Oxford University Press.

Kieser, A. (1989): Organizational, Institutional, and Societal Evolution. Medieval Craft Guilds and the Genesis of Formal Organizations. Administrative Science Quarterly 34, S. 540-564.

Kieserling, A. (1993): Konturen einer soziologischen Unternehmensberatung. Bielefeld: unveröff. Ms.

Looss, W. (1997): Unter vier Augen. Coaching für Manager (4. völlig überarb. Aufl.). Landsberg am Lech: Moderne Industrie.

Luhmann, N. (1962): Der neue Chef. Verwaltungsarchiv 53, S. 11-24.

- (1964): Funktionen und Folgen formaler Organisation. Berlin: Duncker \& Humblot.

- (1966): Recht und Automation in der öffentlichen Verwaltung. Eine verwaltungswissenschaftliche Untersuchung. Berlin: Duncker \& Humblot.

- (1967): Soziologische Aufklärung. Soziale Welt 18, S. 97-123.

- (1975a): Funktionale Analyse. Methode und Systemtheorie. In: Bühl, W.L. (Hg.): Funktion und Struktur. Soziologie vor Geschichte. München: Verlag, S. 98-131.

- (1975b): Strukturauflösung durch Interaktion. Ein analytischer Bezugsrahmen. Bielefeld: unveröff. Ms.

- (1977): Probleme eines Parteiprogramms. In: Baier, H. (Hg.): Freiheit und Sachzwang. Beiträge zu Ehren Helmut Schelskys. Opladen: WDV, S. 167-181.

- (1984a): Soziale Systeme. Frankfurt am Main: Suhrkamp.

- (2000): Organisation und Entscheidung. Opladen: WDV.

- (2005): Die Form „Person“. In. Ders. (Hg.): Soziologische Aufklärung 6 (2. Aufl.). Die Soziologie und der Mensch. Opladen: VS-Verlag, S. 137-149

Moldaschl, M. (1994): „Die werden zur Hyäne“. Erfahrungen und Belastungen in neuen Arbeitsformen. In: Moldaschl, M., Schultz-Wild, R. (Hg.): Arbeitsorientierte Rationalisierung. Fertigungsinseln und Gruppenarbeit im Maschinenbau. Frankfurt a.M., New York: Campus, S. 105-150.

Neuberger, O. (1991): Personalentwicklung. Stuttgart: Enke.

Oevermann, U. (1993): Sturktureigenschaften supervisorischer Praxis. Exemplarische Sequenzanalyse des Sitzungsprotokolls der Supervision eines psychoanalytisch orientierten TherapieTeams im Methodenmodell der objektiven Hermeneutik. In: Bardé, B., Mattke, D. (Hg.): Therapeutische Teams. Göttingen: Vandenhoek \& Ruprecht, S. 149-269.

- (2001): Strukturprobleme supervisorischer Praxis. Eine objektiv hermeneutische Sequenzanalyse zur Überprüfung der Professionalisierungstheorie. Frankfurt a.M.: Humanities Online. 
Pühl, H. (2000): Einzel-Supervision - Coaching - Leitungsberatung: Drei Begriffe für dieselbe Sache. In: Ders. (Hg.): Handbuch der Supervision (2. überarb. Aufl.). Berlin: Edition Marhold, S. 100-111.

Rauen, C. (2000): Glossar. ln: Ders. (Hg.): Handbuch Coaching. Göttingen: Verlag für Angewandte Psychologie, S. 303-308.

Schmidt-Lellek, C.J. (2003): Coaching und Psychotherapie - Differenz und Konvergenz. OSC 10 (3), 227-234.

Schreyögg, A. (1995): Coaching. Eine Einführung für Praxis und Ausbildung. Frankfurt a.M., New York: Campus.

- (2002): Supervision: ein kritischer Blick aus Sicht des Coaches. In: Kessel, L. van, Fellermann, J. (Hg.): Supervision und Coaching in europäischer Perspektive. Beiträge der ANSEKonferenz 2000. Köln: AS Agentur für Supervision GmbH, S. 40-44.

- (2003): Die Differenzen zwischen Supervision und Coaching. OSC 10, S. 217-226.

- (2005): Coaching. In: Mohe, M. (Hg.): Innovative Beratungskonzepte. Ansätze, Fallbeispiele, Reflexionen. Leonberg: Rosenberg, S. 159-178.

Schwertfeger, B. (1998): Der Griff nach der Psyche. Frankfurt a.M., New York: Campus.

Tacke, V. (2000): Netzwerk und Adresse. Soziale Systeme 6, S. 291-320.

Taffertshofer, A. (2006): Coaching in Arbeitsorganisationen. Zu Mode und Praxis einer persönlichen Beratungsmethode. München: Diplomarbeit am Institut für Soziologie der Uni München.

Veiel, A., Schade, J.T. (2002): Black Box BRD. Film.

Voß, G.G., Pongratz, H.J. (1998a): Der Arbeitskraftunternehmer. Eine neue Grundform der Ware Arbeitskraft? Kölner Zeitschrift für Soziologie und Sozialpsychologie 50, S. 131-158.

Weber, M. (1972): Wirtschaft und Gesellschaft. Grundriß der verstehenden Soziologie. Köln: Kiepenheuer \& Witsch.

Weigand, W. (2002): Coaching: eine Methode - Supervision: ein Konzept. In: Kessel, L. van, Fellermann, J. (Hg.): Supervision und Coaching in europäischer Perspektive. Beiträge der ANSE-Konferenz 2000. Köln: AS Agentur für Supervision GmbH, S. 45-54.

Weltz, F. (1986): Aus Schaden dumm werden. Zur Lernschwäche von Verwaltungen. Office Management 34, S. 532-534.

Whyte, W.H. (1956): The Organization Man. New York: Simon and Schuster.

Der Autor: Prof. Dr. Stefan Kühl, geb. 1966, Professor für Soziologie an der Universität der Bundeswehr Hamburg. Lehr- und Forschungsgebiete: Organisationssoziologie, Techniksoziologie, Industrie- und Arbeitssoziologie, Professionssoziologie, Wissenschaftsgeschichte, Gesellschaftstheorie. Anschrift: Universität der Bundeswehr Hamburg, Institut für Gesellschaftswissenschaften, Holstenhofweg 85, 22043 Hamburg. E-Mail: stefan.kuehl@hsu-hh.de,www.hsu-hh.de/kuehl. 